\title{
ON NONLINEAR PERTURBATIONS OF THE EIGEN- VALUES OF A COMPACT SELF-ADJOINT OPERATOR
}

BY MELVYN S. BERGER

\author{
Communicated by Jürgen Moser, May 17, 1967
}

The perturbation theory for an isolated eigenvalue $\lambda_{n}$ of a selfadjoint operator $L$ by a linear self-adjoint operator $T_{\theta}$, depending analytically or continuously on a parameter $\theta$, has been successfully studied since the fundamental results of $F$. Rellich [10]. In this note we state conditions on nonlinear perturbations $N(u)$ that guarantee the validity of an analogue of Rellich's results. We then show how these results can be applied to qualitative problems in the study of real solutions of nonlinear elliptic partial differential equations and periodic solutions of autonomous ordinary differential equations.

Our study is based on focussing attention on a set of nonlinear invariants for the perturbation problem. These invariants can be considered as a set of critical points of a functional defined on a one parameter family of Hilbert manifolds without boundary $\partial A_{R}$.

The critical points are formulated in terms of the LjusternikSchnirelmann category as in Palais [9]. We then show that these critical points are stable under the nonlinear perturbation considered.

Previous studies of such nonlinear problems date back to $\mathrm{E}$. Schmidt [11]. Subsequent extensions were made by A. Hammerstein in [5], J. Cronin [4] and R. Bartle [1] among others. Topological methods for such problems were introduced by L. Ljusternik [8], J. Leray and J. Schauder [7], and M. Krasnoselskii [6]. By narrowing the class of perturbations considered, we are able to obtain somewhat sharper results than these previous treatments. This research was partially supported by N.S.F. grant GP 3904 and U. S. Army (Durham) DA-ARO 31-124-G 156. The author is grateful to Professor $\mathrm{J}$. Moser for helpful conversations in connection with this work.

1. Formulation of the problem. Let $L$ be a positive compact selfadjoint operator mapping a real Hilbert space $H$ into itself. Then the operator equation $u=\lambda L u$ has a countable number of eigenvalues $\lambda_{n}$, each with finite multiplicity. Furthermore the following characterization of $\lambda_{n}$ is valid,

$$
\lambda_{n}^{-1}=\max _{\left[T^{\prime}\right]_{n}} \min _{T^{\prime}}(L u, u)
$$


where $T^{\prime}$ is an $n$-dimensional subspace with elements $u$, normalized by $\|u\|=1 .\left[T^{\prime}\right]_{n}$ is the class of all such subspaces $T^{\prime} \subset H$ for fixed integral $n$. We consider the effect on $\lambda_{n}$ of a perturbation by an operator $N(u)$, not necessarily linear. Thus we consider the operator equation $u=\lambda(L u+N u)$ in the vicinity of $\lambda=\lambda_{n}$ with $\|u\|$ sufficiently small. Here we consider $N(u)$, as depending on the parameter $\|u\|$.

2. Variational operators with symmetry. It is well known, even in the linear case, that some restrictions must be imposed on the perturbation $N u$ to guarantee the validity of an analogue of Rellich's results. The major restrictions we impose are that the operator $N(u)$ should be the gradient of some weakly continuous functional $F(u)$ and that $F(u)$ should be invariant under some group $G$. For linear problems this restricts the perturbations considered to be self-adjoint, and the associated quadratic functional is automatically invariant under the group $Z_{2}$ generated by the antipodal map in $H$. For simplicity, we restrict attention to nonlinear problems invariant under the group $Z_{2}$. More precisely, we assume:

(i) $\|N u-N v\| \leqq k\left\{\|u\|^{p-1}+\|v\| \|^{p-1}\right\}\|u-v\|$ where $p, k$ are constants independent of $u, v$ for $\|u\|,\|v\|$ sufficiently small $p \geqq 1$,

(ii) $N(-u)=-N(u)$,

(iii) $N$ maps weakly convergent sequences into strongly convergent sequences.

(iv) $\int_{0}^{1}(u, N(s u)) d s-\int_{0}^{1}(v, N(s v)) d v=\int_{0}^{1}(u-v, N(s u+(1-s) v)) d s$. (Condition (iv) states that $N$ is a gradient operator.)

\section{Results in Hilbert space.}

THEOREM 1. Let the operator $N(u)$ satisfy conditions (i)-(iv) of $\$ 2$. Suppose $\lambda_{n}$ is an eigenvalue of multiplicity $p$ of the operator equation $u=\lambda L u$. Then the operator equation $u=\lambda(L u+N u)$ has at least $p$ one parameter families of nontrivial solutions $u_{n}(R), u_{n+1}(R), \cdots$, $u_{n+p-1}(R)$, for sufficiently small $R$, where $\frac{1}{2}\|u\|^{2}=R$, in the vicinity of $\lambda=\lambda_{n}$. Each solution can be characterized as a solution of a variational problem,

$$
c_{n}(R)=\sup _{[T]_{n}} \min _{T}\left[\frac{1}{2}(L u, u)+\int_{0}^{1}(u, N(s u)) d s\right],
$$

where $[T]_{n}$ is an isotopy class of sets on $\partial \sum_{R}=\left\{u /\|u\|^{2}=2 R\right\}$. Furthermore as $R \rightarrow 0, \lambda_{n}(R) \rightarrow \lambda_{n}$.

REMARK 1. A similar result is valid for the operator equation of the form $u+N u=\lambda L u$, as in [2]. 
REMARK 2. The classes $[T]_{n}$ are defined as in [3], by the Ljusternik-Schnirelmann category on $\partial \sum_{R} / Z_{2}$.

4. Application to nonlinear elliptic partial differential equations. Let $G$ be a bounded domain in real $n$-dimensional Euclidean space $R^{n}$ with boundary $\partial G$. Let $D_{j}=\partial / \partial x_{j}, 1 \leqq j \leqq n, D^{\alpha}=\prod_{j} D_{j}^{\alpha}$ with $|\alpha|$ $=\alpha_{1}+\alpha_{2} \cdots+\alpha_{n}$. Consider the Hilbert spaces

$$
\begin{aligned}
& W_{m, 2}(G)=\left\{u\left|D^{\alpha} u \in L_{2}(G),\right| \alpha \mid \leqq m\right\}, \\
& W_{m, 2}(G)=\text { closure of } C_{0}^{\infty}(G) \text { in } W_{m, 2}(G) .
\end{aligned}
$$

In this context, Theorem 1 can be reformulated as a result on the bifurcation theory of elliptic partial differential equations. Indeed, if we consider a formally self-adjoint uniformly elliptic differential operator of order $2 m$

$$
A u=\sum_{|\alpha|,|\beta| \leqslant m} D^{\alpha}\left(a_{\alpha \beta}(x) D^{\beta} u\right)
$$

and an analogous operator $B u$ of order $2 m-2$, we may study the following eigenvalue problems:

$$
A u=\lambda(B u+N u),\left.\quad D^{\alpha} u\right|_{\partial G}=0, \quad|\alpha| \leqq m-1,
$$

where

$$
\begin{gathered}
N u=\sum_{|\alpha| \leqq m-1}(-1)^{|\alpha|} D^{\alpha}\left(A_{\alpha}\left(x, u, \cdots, D^{m-1} u\right)\right), \\
(-1)^{|\alpha|} D^{\alpha}\left(A_{\alpha}\left(x, u, \cdots, D^{m-1} u\right)\right)=\frac{\partial}{\partial p_{\alpha}} \tilde{A}\left(x, u, \cdots, D^{m-1} u\right)
\end{gathered}
$$

with $\tilde{A}\left(x,-u, \cdots,-D^{m-1} u\right)=\tilde{A}\left(x, u, \cdots, D^{m-1} u\right)$.

$$
A u=\lambda B u, \quad D^{\alpha} u_{\mid \partial G}=0, \quad|\alpha| \leqq m-1 .
$$

We consider weak solutions of (3) in the Hilbert space $\dot{W}_{m, 2}(G)$. Under appropriate growth restrictions on the nonlinear term $N u$ (cf. [3]) and positivity conditions on the Dirichlet forms associated with $A$ and $B$ these weak solutions may be formulated in terms of an operator equation of the form $u=\lambda(L u+N u)$. Thus Theorem 1 applies directly to yield

TheOREM 2. From each eigenvalue $\lambda_{n}$ of the linearized equation $\left(3^{\prime}\right)$ of multiplicity $p$, there bifurcates at least $p$ one parameter families of real solutions of the nonlinear equation (3). These solutions are smooth provided the coefficients of the equation and $\partial G$ are sufficiently smooth.

(The smoothness of the solutions mentioned in Theorem 2 is a consequence of $L_{p}$ regularity for linear elliptic systems.) 
5. Application to periodic solutions of autonomous systems of ordinary differential equations. We consider the equation

$$
\ddot{\mathrm{x}}=A \mathrm{x}+f(\mathrm{x})
$$

where $\mathrm{x}=\left(x_{1}, \cdots, x_{n}\right)$ is a real $n$-vector of functions $x_{i}(t), A$ is a constant positive definite self-adjoint matrix and $f(x)$ is an $n$-vector of its functions, holomorphic in some neighborhood of the origin beginning with quadratic terms in $x$. We assume $f(x)$ is the gradient of some scalar function $F\left(x_{1}, \cdots, x_{n}\right)$ and that $f(-x)=-f(x)$. Furthermore we impose the following condition on the eigenvalues of $A$ :

Assumption N.D. If the eigenvalues of the matrix $A$ are denoted $\lambda_{1}^{2}, \lambda_{2}^{2}, \cdots, \lambda_{n}^{2}$, then no ratio of the form $\lambda_{j}^{2} / \lambda_{k}^{2}$ should equal an integer for $j, k=1,2, \cdots, n(j \neq k)$.

Thus the following result of Lyapunov is a consequence of Theorem 1.

THEOREM 3. Under the above assumptions on the matrix $A$ and the vector function $\boldsymbol{f}(\boldsymbol{x})$, the equation (4) has at least $n$ real one parameter families of nonzero periodic solutions. Furthermore if the minimal period of the jth family is denoted by $T_{j}(R)$, then $\lim _{R \rightarrow 0} T_{j}(R)=2 \pi \lambda_{j}$.

For an analytical proof of this result, independent of the assumption $f(-x)=-f(x)$, we refer to the book of C. L. Siegel [13]. The relevance of this parity assumption for the problem at hand is the following:

CONJECTURE. Theorem 3 is valid independent of the assumption N.D.

\section{BIBLIOGRAPHY}

1. R. Bartle, Singular points of functional equations, Trans. Amer. Math. Soc. 75 (1953), 366-384.

2. M. Berger, An application of the calculus of variations on the large to the equations of nonlinear elasticity, Bull. Amer. Math. Soc. 73 (1967), 520-525.

3. - A Sturm-Liouville theorem for nonlinear elliptic partial differential equations, Ann. Scuola di Pisa 20 (1966), 543-582.

4. J. Cronin, Branch points of solution of equations in Banach space. I, II, Trans. Amer. Math. Soc. 69 (1950), 208-231; 76 (1954), 207-222.

5. A. Hammerstein, Nichtlineare Integralgleichungen nebst Anwendungen, Acta Math. 54 (1930), 117-176.

6. M. KrasnoselskYi, Application of variational methods to the problem of bifurcation points, Math. Sb. 33 (1953), 199-214.

7. J. Leray and J. Schauder, Topologie et equations fonctionelles, Ann. Sci. Ecole Norm. 51 (1934), 45-78.

8. L. Ljusternik, Topologische Grundlagen der allgemeinen Eigenwerttheorie, Monat. Math. Phys. (1) 37 (1930), 125-130. 
9. R. Palais, Ljusternik-Schnirelmann theory on Banach manifolds, Topology 15 (1966), 115-132.

10. F. Rellich, Störungstheorie der Spektralzerlegung. I, Math. Annalen 113 (1936), 600-619.

11. E. Schmidt, Zur Theorie der linearen und nichtlinearen Integralgleichungen. III, Teil, Math. Ann. 65 (1910), 370-399.

12. J. Schwartz, Generalizing the Ljusternik-Schnirelmann theory of critical points, Comm. Pure Appl. Math. 27 (1964), 307-315.

13. C. L. Siegel, Vorlesungen ïber Himmelsmechanik, Springer-Verlag, Berlin, 1965.

Courant Institute of Mathematical Sciences, New York University

\title{
EXTREMAL PROBLEMS FOR FUNCTIONS OF BOUNDED BOUNDARY ROTATION ${ }^{1}$
}

\author{
BY BERNARD PINCHUK ${ }^{2}$
}

Communicated by Alberto P. Calderon, April 19, 1967

1. Preliminaries. Let $V_{k}$ denote the class of analytic functions in $D=\{z:|z|<1\}$ which have there the representation

$$
f(z)=\int_{0}^{z} \exp \left(-\int_{0}^{2 \pi} \log \left(1-\zeta e^{-i \theta}\right) d \psi(\theta)\right) d \zeta
$$

where $\psi(\theta)$ is a real valued function of bounded variation for $0 \leqq \theta$ $<2 \pi$, satisfying there the conditions

$$
\int_{0}^{2 \pi} d \psi(\theta)=2, \quad \int_{0}^{2 \pi}|d \psi(\theta)| \leqq k .
$$

$V_{k}$ is the class of analytic functions in $D$ which have boundary rotation bounded by $k \pi$. Thus, $V_{k}$ consists of those functions $f(z)$ $=z+a_{2} z^{2}+\cdots$ which are analytic and satisfy $f^{\prime}(z) \neq 0$ in $D$, and map $D$ onto a domain having boundary rotation bounded by $k \pi$.

Briefly, the boundary rotation of a schlicht domain $G$ with continuously differentiable boundary curve is the total variation of the direc-

1 Research partially sponsored by the Air Force Office of Scientific Research, Office of Aerospace Research, U. S. Air Force, under AFOSR Grant No. AF-AFOSR 1077-66.

2 The author wishes to express his gratitude to Professor Harry E. Rauch for his many helpful conversations during this research. 